Kragujevac Journal of Mathematics

Volume 46(1) (2022), Pages 49-64.

\title{
SOLUTION SET FOR IMPULSIVE FRACTIONAL DIFFERENTIAL INCLUSIONS
}

\author{
MOUSTAFA BEDDANI ${ }^{1}$
}

\begin{abstract}
This paper aims to an initial value problem for an impulsive fractional differential inclusion with the Riemann-Liouville fractional derivative. We apply Covitz and Nadler theorem concerning the study of the fixed point for multivalued maps to obtain the existence results for the given problems. We also obtain some topological properties about the solution set.
\end{abstract}

\section{INTRODUCTION}

We study the existence of solutions and determine certain topological properties of the solutions set for the following impulsive fractional differential inclusion:

$$
\left\{\begin{array}{l}
{ }^{R L} D^{\alpha} y(t) \in F(t, y(t)) \quad \text { a.e. } t \in J=(0, T], t \neq t_{k}, \\
\lim _{t \longrightarrow 0^{+}} t^{1-\alpha} y(t)=c \\
\left.\Delta^{*} y\right|_{t_{k}}=I_{k}\left(y\left(t_{k}^{-}\right)\right),
\end{array}\right.
$$

where $k=1, \ldots, m, 0<\alpha \leq 1,{ }^{R L} D^{\alpha}$ is the Riemann-Liouville fractional derivative, $F: J \times \mathbb{R} \rightarrow\{X \subset \mathbb{R}: X \neq \emptyset\}$ is a given multivalued function, $c \in \mathbb{R}, I_{k}: \mathbb{R} \rightarrow \mathbb{R}$ are continuous functions, $0=t_{0}<t_{1}<\cdots<t_{m}<t_{m+1}=T$ and $\left.\Delta^{*} y\right|_{t_{k}}=y^{*}\left(t_{k}^{+}\right)-y\left(t_{k}^{-}\right)$, where $y^{*}\left(t_{k}^{+}\right)=\lim _{t \rightarrow t_{k}^{+}}\left(t-t_{k}\right)^{1-\alpha} y(t)$ and $y\left(t_{k}^{-}\right)=\lim _{t \rightarrow t_{k}^{-}} y(t)$.

More precisely, we present an overall existence result for (1.1) by using Covitz and Nadler fixed-point theorem for multivalued maps. Afterwards, we prove the compactness and acyclicity of the solution set for this problem.

Key words and phrases. Impulsive fractional differential inclusions, Riemann-Liouville fractional derivative, fixed point, solution set, compactness, contractible.

2010 Mathematics Subject Classification. Primary: 34A60, 34A08 Secondary: 34A37.

DOI 10.46793/KgJMat2201.049B

Received: March 25, 2019.

Accepted: August 19, 2019. 
Since the 1960s, the subject of functional differential inclusions and impulsive ordinary differential inclusions with various conditions have been investigated by many authors $[1,2,8,11,14,24-26,32,35,39,40]$, and has several applications in different areas as engineering, electrical, networks electrochemistry, fluid flow, etc. For more details we refer the reader to see the following references $[4,13,20,21,30,31,34,36]$.

The topological and geometric properties of the solution set for differential inclusions are examined by many mathematicians (see for example $[3,10,16,17,22,37]$ ) where the concept of quasi-concavity is extended to multifunctions contractibility, absolutely retract, acyclicity, $R_{\delta}$-sets properties are given.

This work is structured as follows: in the second section, we recall some definitions and properties that are needed throughout this article. Afterwards, in the third section, we show that the solution sets is contractible to a point. Finally, we give an example which illustrate the principle result of this paper.

\section{Preliminary Results}

The object of this section is to recall some basic definitions and useful notations in multivalued analysis. Let $C([a, b], \mathbb{R})$ be the Banach space of all continuous functions $h$ from the interval $[a, b]$ into $\mathbb{R}$ with the norm

$$
\|h\|_{\infty}=\sup _{t \in[a, b]}|h(t)|,
$$

and $L^{1}([a, b], \mathbb{R})$ the Banach space of all Lebesgue integrable functions $h$ from the interval $[a, b]$ into $\mathbb{R}$ with the norm

$$
\|h\|_{L^{1}}=\int_{a}^{b}|h(t)| d t .
$$

For a given metric space $E$, we denote:

- $\mathcal{P}(E)=\{X \subset E: X \neq \emptyset\}$;

- $\mathcal{P}_{c l}(E)=\{X \in \mathcal{P}(E): X$ is closed $\}$

- $\mathcal{P}_{b}(E)=\{X \in \mathcal{P}(E): X$ is bounded $\}$;

- $\mathcal{P}_{c p}(E)=\{X \in \mathcal{P}(E): X$ is compact $\}$;

- $\mathcal{P}_{c}(E)=\{X \in \mathcal{P}(E): X$ is convex $\}$;

- $\mathcal{P}_{c p, c}(E)=\mathcal{P}_{c p}(E) \cap \mathcal{P}_{c}(E)$.

If $X$ and $Y$ are two subsets of the metric space $E$, and $x$ (resp. $y$ ) is a point in $X$ (resp. $Y$ ), we denote:

$$
d(x, Y)=\inf _{y \in Y} d(x, y) \quad \text { and } \quad d(X, y)=\inf _{x \in X} d(x, y) .
$$

Recall that, the Hausdorff pseudo-metric distance $H_{d}$ on $\mathcal{P}(E)$ defined by

$$
H_{d}(X, Y):=\max \left\{\sup _{x \in X} d(x, Y), \sup _{y \in Y} d(X, y)\right\} .
$$


Form the previous definition, it is well-known that $\left(\mathcal{P}_{c l, b}(E), H_{d}\right)\left(\operatorname{resp} .\left(\mathcal{P}_{c l}(E), H_{d}\right)\right)$ is a metric space (resp. is a generalized metric space).

Definition 2.1. Let $M: \rightarrow \mathcal{P}(E)$ be a multivalued map.

(a) We say that $M$ is $\gamma$-Lipschitz if there exists a positive real number $\gamma$ such that

$$
H_{d}(M(x), M(y)) \leq \gamma d(x, y), \quad \text { for all } x, y \in E .
$$

(b) The map $M$ is called a contraction if it is $\gamma$-Lipschitz for some $0<\gamma<1$.

Notice that, if $M$ is $\gamma$-Lipschitz on a Banach space $E$, then for every real number $\gamma^{\prime}$ greater than $\gamma, M(x) \subset M(y)+\gamma^{\prime} d(x, y) B(0,1)$, where $B(0,1)$ is the unit ball of $E$.

Definition 2.2. Let $G: X \rightarrow \mathcal{P}_{c l}(Y)$ be a multivalued map, where $X$ and $Y$ are two metric spaces.

(a) We say that $G$ is closed valued (resp. convex valued) if $G(x)$ is closed (resp. convex) for all $x \in E$.

(b) Every single-valued map $g: X \rightarrow Y$ is called a selection of $G$. We write $g \subset G$ whenever $g(x) \in G(x)$ for all $x \in X . G: J \times \mathbb{R} \rightarrow \mathcal{P}(\mathbb{R})$, we define the set of selections of $G$ by

$$
S_{G, x}=\left\{v \in L^{1}(J, \mathbb{R}): v(t) \in G(t, x(t)) \text { a.e. } t \in J\right\} .
$$

Definition 2.3. A multivalued map $G: J \times \mathbb{R} \rightarrow \mathcal{P}(\mathbb{R})$ is called $L^{1}$-Carathéodory if the following are satisfied:

(a) the function $G(\cdot, x)$ is measurable for each $x \in \mathbb{R}$;

(b) the function $G(t, \cdot)$ is upper semi-continuous for almost all $t \in J$;

(c) for every positive real $r$, there exists a function $f_{r} \in L^{1}\left(J, \mathbb{R}_{+}\right)$such that

$\|G(t, x)\|=\sup \{|v|: v \in G(t, x)\} \leq f_{r}(t) \quad$ a.e. $t \in J$ and for all $x \in[-r, r]$.

For more details about multivalued analysis, we refer the reader to see $[5-7,12,23$, 27-29].

Below we present the definition of contractible spaces, and for details about this type of spaces, we recommend $[5,10,15,33]$.

Definition 2.4. A contractible subset of a Banach space $X$ is a nonempty subset $A$ of $X$ for which there exists a continuous homotopy $\psi: A \times[0,1] \rightarrow A$ and $a_{0} \in A$ such that for all $a \in A, \psi(a, 0)=a$ and $\psi(a, 1)=a_{0}$.

We give now two basic definitions used frequently in fractional computation theory. Definition 2.5 ([18,19]). Let $h \in L^{1}\left([a, b], \mathbb{R}_{+}\right)$. The fractional order integral of $h$ is given by

$$
I_{a}^{\alpha} h(t)=\int_{a}^{t} \frac{(t-s)^{\alpha-1}}{\Gamma(\alpha)} h(s) d s .
$$


Definition 2.6 ([19]). Let $b$ be a positive real number and $h$ a real function defined on the interval $[0, b]$. The Riemann-Liouville fractional derivative of order $\alpha \in \mathbb{R}_{+}$of $h$ is defined as follows:

$$
{ }^{R L} D_{0^{+}}^{\alpha} h(t)=\frac{1}{\Gamma(n-\alpha)} \frac{d^{n}}{d t^{n}}\left(\int_{0}^{t}(t-s)^{n-\alpha-1} h(s) d s\right) .
$$

\section{MAin Results}

Let

$$
\begin{aligned}
P C_{*}([0, T], \mathbb{R})= & \left\{y:[0, T] \longrightarrow \mathbb{R}: y_{k} \in C\left(t_{k}, t_{k+1}\right], k=0, \ldots, m\right. \text {, and there } \\
& \text { exist } \left.y\left(t_{k}^{-}\right), y_{*}\left(t_{k}^{+}\right), k=1, \ldots, m \text {, with } y\left(t_{k}\right)=y\left(t_{k}^{-}\right)\right\} .
\end{aligned}
$$

It is known that this set is Banach space with the norm

$$
\|y\|_{P C_{*}}=\max _{k=1, \ldots m}\left\|y_{k}\right\|_{*},
$$

where $y_{k}$ is the restriction of $y$ to the interval $J_{k}=\left(t_{k}, t_{k+1}\right]$ for every $k=0, \ldots, m$, and

$$
\left\|y_{k}\right\|_{*}=\sup _{t \in\left[t_{k}, t_{k+1}\right]}\left|\left(t-t_{k}\right)^{1-\alpha} y_{k}(t)\right|, \quad \text { for every } k=1, \ldots, m .
$$

When $A \subset P C_{*}([0, T], \mathbb{R})$, we define $\mathcal{A}_{\alpha}$ by

$$
\mathcal{A}_{\alpha}=\left\{y_{\alpha}: y \in A\right\}
$$

where

$$
y_{\alpha}(t)= \begin{cases}\left(t-t_{k}\right)^{1-\alpha} y(t), & \text { if } t \in\left(t_{k}, t_{k}+1\right], \\ \lim _{t \rightarrow t_{k}}\left(t-t_{k}\right)^{1-\alpha} y(t), & \text { if } t=t_{k} .\end{cases}
$$

Theorem 3.1. Let $A$ be a bounded subset in the Banach space $P C_{*}([0, T], \mathbb{R})$ such that $\mathcal{A}_{\alpha}$ is equicontinuous on $P C([0, T], \mathbb{R})$. Then the set $A$ is relatively compact in $P C_{*}([0, T], \mathbb{R})$.

Proof. We know that, if $\left\{y_{n}\right\}_{n=1}^{\infty} \subset A$, then $\left\{\left(y_{\alpha}\right)_{n}\right\}_{n=1}^{\infty} \subset P C([0, T], \mathbb{R})$. From ArzelaAscoli theorem, the set $K_{0}=\left\{\left(y_{\alpha}\right)_{n}: n \in \mathbb{N}^{*}\right\}$ is relatively compact in the space $P C([0, T], \mathbb{R})$. So, we can find a subsequence of $\left(y_{\alpha}\right)_{n \in \mathbb{N}}$ still denoted by the same indices such that $\lim y_{n} \in\left(P C([0, T], \mathbb{R}),\|\cdot\|_{P C}\right)$. Put $y=\lim y_{n}$. We have

$$
\left.\left\|\left(y_{\alpha}\right)_{n}-y\right\|_{*}=\sup _{t \in\left[t_{k}, t_{k+1}\right]}\left(t-t_{k}\right)^{1-\alpha} \mid y_{\alpha}\right)_{n}(t)-y(t) \mid \rightarrow 0, \quad n \rightarrow+\infty .
$$

So, $y_{n} \rightarrow y, n \rightarrow+\infty$, on $P C_{*}([0, T], \mathbb{R})$.

To explain our results, we need the following lemmas. 
Lemma $3.1([38])$. Let $\alpha$ be a positive real number and $n=1+[\alpha]$. Then the following differential equation ${ }^{R L} D_{a^{+}}^{\alpha} h(t)=0$, has solutions of the form $h(t)=\sum_{k=1}^{n} c_{k}(t-a)^{\alpha-k}$ for some real numbers $c_{1}, c_{2}, \ldots, c_{n}$.

Lemma $3.2([38])$. Let $\alpha$ be a positive real number and $n=1+[\alpha]$. Then there exist some real numbers $c_{1}, c_{2}, \ldots, c_{n}$ such that

$$
I^{\alpha R L} D_{a^{+}}^{\alpha} h(t)=h(t)+\sum_{k=1}^{n} c_{k}(t-a)^{\alpha-k} .
$$

Lemma 3.3. Let $\alpha$ be a real number in the interval $(0,1)$ and $h$ a continuous function. If $y$ is a solution of following problem

$$
\begin{aligned}
{ }^{R L} D^{\alpha} y(t) & =h(t), \quad \text { for all } t \in J \text { and } t \neq t_{m} \text { for all } k=1, \ldots, m, \\
\left.\Delta^{*} y\right|_{t_{k}} & =I_{k}\left(y\left(t_{k}^{-}\right)\right), \quad \text { for all } k=1, \ldots, m, \\
\lim _{t \rightarrow 0} t^{1-\alpha} y(t) & =c .
\end{aligned}
$$

Then

$$
y(t)= \begin{cases}t^{\alpha-1} c+\frac{1}{\Gamma(\alpha)} \int_{0}^{t}(t-s)^{\alpha-1} h(s) d s, & \text { if } 0 \leq t \leq t_{1}, \\ \left(t-t_{1}\right)^{\alpha-1} t_{1}^{\alpha-1} c+\frac{\left(t-t_{1}\right)^{\alpha-1}}{\Gamma(\alpha)} \int_{0}^{t_{1}}\left(t_{1}-s\right)^{\alpha-1} h(s) d s & \\ +\frac{\left(t-t_{1}\right)^{\alpha-1}}{\Gamma(\alpha)} I_{1}\left(y\left(t_{1}^{-}\right)\right)+\frac{1}{\Gamma(\alpha)} \int_{t_{1}}^{t}(t-s)^{\alpha-1} h(s) d s, & \text { if } t_{1}<t \leq t_{2}, \\ \left(t-t_{k}\right)^{\alpha-1} \prod_{i=1}^{k}\left(t_{i}-t_{i-1}\right)^{\alpha-1} c & \\ +\frac{\left(t-t_{k}\right)^{\alpha-1}}{\Gamma(\alpha)}\left[\int_{t_{k-1}}^{t_{k}}\left(t_{k}-s\right)^{\alpha-1} h(s) d s+\right. & \\ \left.\sum_{i=1}^{k-1} \prod_{j=1}^{k-i}\left(t_{k-j+1}-t_{k-j}\right)^{\alpha-1} \int_{t_{i-1}}^{t_{i}}\left(t_{i}-s\right)^{\alpha-1} h(s) d s\right] & \\ +\frac{\left(t-t_{k}\right)^{\alpha-1}}{\Gamma(\alpha)}\left[I_{k}\left(y\left(t_{k}^{-}\right)\right)\right. & \\ \left.+\sum_{i=1}^{k-1} \prod_{j=1}^{k-i}\left(t_{k-j+1}-t_{k-j}\right)^{\alpha-1} I_{i}\left(y\left(t_{i}^{-}\right)\right)\right] & \\ +\frac{1}{\Gamma(\alpha)} \int_{t_{k}}^{t}(t-s)^{\alpha-1} h(s) d s, & \text { if } t_{k}<t \leq t_{k+1}, \\ & 2 \leq k \leq m .\end{cases}
$$

Proof. Suppose that $y$ satisfies (3.1)-(3.3). It is clear when $0 \leq t \leq t_{1}$, we have

$$
{ }^{R L} D^{\alpha} y(t)=h(t) .
$$


From the previous lemma, we get

$$
y(t)=t^{\alpha-1} c_{1}+\frac{1}{\Gamma(\alpha)} \int_{0}^{t}(t-s)^{\alpha-1} h(s) d s .
$$

Hence, $c_{1}=c$. Thus,

$$
y(t)=t^{\alpha-1} c+\frac{1}{\Gamma(\alpha)} \int_{0}^{t}(t-s)^{\alpha-1} h(s) d s .
$$

When $t_{1}<t \leq t_{2}$, we can obtain, by using the previous lemma, that

$$
\begin{aligned}
y(t)= & \left(t-t_{1}\right)^{\alpha-1} y^{*}\left(t_{1}^{+}\right)+\frac{1}{\Gamma(\alpha)} \int_{t_{1}}^{t}(t-s)^{\alpha-1} h(s) d s \\
= & \left(t-t_{1}\right)^{\alpha-1}\left(I_{1}\left(y\left(t_{1}^{-}\right)+y\left(t_{1}^{-}\right)\right)+\frac{1}{\Gamma(\alpha)} \int_{t_{1}}^{t}(t-s)^{\alpha-1} h(s) d s\right. \\
= & \left(t-t_{1}\right)^{\alpha-1} t_{1}^{\alpha-1} c+\frac{\left(t-t_{1}\right)^{\alpha-1}}{\Gamma(\alpha)} \int_{0}^{t_{1}}\left(t_{1}-s\right)^{\alpha-1} h(s) d s \\
& +\frac{1}{\Gamma(\alpha)} \int_{t_{1}}^{t}(t-s)^{\alpha-1} h(s) d s+\left(t-t_{1}\right)^{\alpha-1} I_{1}\left(y\left(t_{1}^{-}\right)\right) .
\end{aligned}
$$

If $t_{2}<t \leq t_{3}$, Lemma 3.2 implies

$$
\begin{aligned}
y(t)= & \left(t-t_{2}\right)^{\alpha-1} y^{*}\left(t_{2}^{+}\right)+\frac{1}{\Gamma(\alpha)} \int_{t_{2}}^{t}(t-s)^{\alpha-1} h(s) d s \\
y(t)= & \left(t-t_{2}\right)^{\alpha-1}\left[y\left(t_{2}^{-}\right)+I_{2}\left(y\left(t_{2}^{-}\right)\right)\right]+\frac{1}{\Gamma(\alpha)} \int_{t_{2}}^{t}(t-s)^{\alpha-1} h(s) d s \\
= & \left(t-t_{2}\right)^{\alpha-1}\left(t_{2}-t_{1}\right)^{\alpha-1} t_{1}^{\alpha-1}+\frac{\left(t-t_{2}\right)^{\alpha-1}\left(t_{2}-t_{1}\right)^{\alpha-1}}{\Gamma(\alpha)} \int_{0}^{t_{1}}\left(t_{1}-s\right)^{\alpha-1} h(s) d s \\
& +\frac{\left(t-t_{2}\right)^{\alpha-1}}{\Gamma(\alpha)} \int_{t_{1}}^{t_{2}}\left(t_{2}-s\right)^{\alpha-1} h(t) d s+\frac{1}{\Gamma(\alpha)} \int_{t_{2}}^{t}(t-s)^{\alpha-1} h(s) d s \\
& +\left(t-t_{2}\right)^{\alpha-1}\left[\left(t_{2}-t_{1}\right)^{\alpha-1} I_{1}\left(y\left(t_{1}^{-}\right)\right)+I_{2}\left(y_{2}\left(t_{2}^{-}\right)\right)\right] .
\end{aligned}
$$

Finally, when $t_{k}<t \leq t_{k+1}$, we obtain (3.4), by using Lemma 3.2.

Definition 3.1. Let $y$ be a function in $P C_{*}$. We say that $y$ is a mild solution of the problem (1.1) if there exists $v \in L^{1}(J, \mathbb{R})$ such that $v(t) \in F(t, y(t))$ a.e. on $J \backslash$ $\left\{t_{1}, \ldots, t_{m}\right\}, \lim _{t \longrightarrow 0} t^{1-\alpha} y(t)=c,\left.\Delta^{*} y\right|_{t_{k}}=I_{k}\left(t_{k}^{-}\right)$for all $k=1, \ldots, m$, and ${ }^{R L} D^{\alpha} y(t)=$ $v(t)$ for all $t \in J \backslash\left\{t_{1}, \ldots, t_{m}\right\}$.

Definition $3.2([6,23])$. A single-valued map $f:[0, a] \times X \rightarrow Y$ be a single-valued map is said to be measurable locally Lipschitz (mLL) if

(1) $f(\cdot, x)$ is measurable for every $x \in X$, and

(2) for each $x \in X$, there exists a neighborhood $V_{x}$ of $x$ and an integrable function $L_{x}:[0, a] \rightarrow[0, \infty)$ such that

$$
d^{\prime}\left(f\left(t, x_{1}\right), f\left(t, x_{2}\right)\right) \leq L_{x}(t) d\left(x_{1}, x_{2}\right) \quad \text { a.e. } t \in[0, a] \text { and } x_{1}, x_{2} \in V_{x} .
$$


Definition 3.3 ([6, 23]). A mapping $F:[0, a] \times X \rightarrow \mathcal{P}(Y)$ is mLL-selectionable provided there exists a measurable locally-Lipchitzian map $f:[0, a] \times X \rightarrow Y$ such that $f \in F$.

Lemma $3.4([2,11])$. If $N: X \rightarrow \mathcal{P}_{c l}(X)$ is a contraction on a complete metric space $X$, then the fixed point set of $N$ is nonempty.

Theorem 3.2. Let $F: J \times \mathbb{R} \rightarrow \mathcal{P}_{c p}(\mathbb{R})$ be a mLL-selectionable multivalued map such that the following properties are satisfied:

$\left(H_{1}\right)$ there exist $\bar{a}$ and $\bar{b}$ in $\mathbb{R}_{+}$such that for every $x \in \mathbb{R}$, we have

$$
\|F(t, x)\|_{\mathcal{P}} \leq \bar{a}|x|+\bar{b} \quad \text { a.e. } t \in J ;
$$

$\left(H_{2}\right)$ there exist $a_{k}$ and $b_{k} \in \mathbb{R}_{+}$such that

$$
\left|I_{k}(x)\right| \leq a_{k}|x|+b_{k}, \quad \text { for } x \in \mathbb{R} ;
$$

$\left(H_{3}\right)$ there exist $p \in C\left([0, T], \mathbb{R}_{+}\right)$such that

$$
H_{d}\left(F\left(t, z_{1}\right), F\left(t, z_{2}\right)\right) \leq p(t)\left\|z_{1}-z_{2}\right\|, \quad \text { for all } z_{1}, z_{2} \in \mathbb{R},
$$

and $d(0, F(t, 0)) \leq p(t), t \in J$;

$\left(H_{4}\right)$ there exist a real number $L \in \mathbb{R}_{+}$such that

$$
\left|I_{k}\left(z_{1}\right)-I_{k}\left(z_{2}\right)\right| \leq L\left\|z_{1}-z_{2}\right\|, \quad \text { for all } z_{1}, z_{2} \in \mathbb{R} .
$$

If

$$
\frac{T^{\alpha}\|p\|_{\infty} \Gamma(\alpha)\left(1+m T^{\alpha-1}\right)}{\Gamma(2 \alpha)}+\frac{m T_{0}^{\alpha-1} L}{\Gamma(\alpha)}<1
$$

then (1.1) has a solution. In addition, if $F: J \times \mathbb{R} \rightarrow \mathcal{P}_{c p}(\mathbb{R})$ is a Carathéodory multivalued map with compact convex values, then the solution set is contractible and compact, and hence it is acyclic.

Proof. Step 1. Existence of solutions. Let $P: P C_{*} \longrightarrow \mathcal{P}\left(P C_{*}\right)$ the operator defined by

$$
\begin{aligned}
P(y)= & \left\{h \in P C_{*}: h(t)=\left(t-t_{k}\right)^{\alpha-1} \prod_{i=1}^{k}\left(t_{i}-t_{i-1}\right)^{\alpha-1} c\right. \\
& +\frac{\left(t-t_{k}\right)^{\alpha-1}}{\Gamma(\alpha)}\left[\int_{t_{k-1}}^{t_{k}}\left(t_{k}-s\right)^{\alpha-1} h(s) d s\right. \\
& \left.+\sum_{i=1}^{k-1} \prod_{j=1}^{k-i}\left(t_{k-j+1}-t_{k-j}\right)^{\alpha-1} \int_{t_{i-1}}^{t_{i}}\left(t_{i}-s\right)^{\alpha-1} v(s) d s\right]+\frac{\left(t-t_{k}\right)^{\alpha-1}}{\Gamma(\alpha)}\left[I_{k}\left(y\left(t_{k}^{-}\right)\right)\right. \\
& \left.\left.+\sum_{i=1}^{k-1} \prod_{j=1}^{k-i}\left(t_{k-j+1}-t_{k-j}\right)^{\alpha-1} I_{i}\left(y\left(t_{i}^{-}\right)\right)\right]+\frac{1}{\Gamma(\alpha)} \int_{t_{k}}^{t}(t-s)^{\alpha-1} v(s) d s\right\},
\end{aligned}
$$

where $v \in S_{F, y}=\left\{v \in L^{1}(J, \mathbb{R}): v(t) \in F(t, y(t))\right.$ a.e. $\left.t \in J\right\}$. Now, we show that the operator $F$ satisfies the hypotheses $\left(H_{1}\right),\left(H_{2}\right)$ and $\left(H_{3}\right)$ of Lemma 3.4. To prove 
that $P(y) \in \mathcal{P}_{c l}\left(P C_{*}([0, T], \mathbb{R})\right)$ for all $y \in P C_{*}([0, T], \mathbb{R})$, let $\left\{u_{n}\right\}_{n=0}^{\infty} \in F(y)$ be a sequence converges to $u$ on the space $P C_{*}([0, T], \mathbb{R})$. Then $u \in P C_{*}([0, T], \mathbb{R})$ and there exists $v_{n} \in S_{F, y}$ such that, for each $t \in(0, T]$

$$
\begin{aligned}
u_{n}(t)= & \left(t-t_{k}\right)^{\alpha-1} \prod_{i=1}^{k}\left(t_{i}-t_{i-1}\right)^{\alpha-1} c \\
& +\frac{\left(t-t_{k}\right)^{\alpha-1}}{\Gamma(\alpha)}\left[\int_{t_{k-1}}^{t_{k}}\left(t_{k}-s\right)^{\alpha-1} v_{n}(s) d s\right. \\
& \left.+\sum_{i=1}^{k-1} \prod_{j=1}^{k-i}\left(t_{k-j+1}-t_{k-j}\right)^{\alpha-1} \int_{t_{i-1}}^{t_{i}}\left(t_{i}-s\right)^{\alpha-1} v_{n}(s) d s\right] \\
& +\frac{\left(t-t_{k}\right)^{\alpha-1}}{\Gamma(\alpha)}\left[I_{k}\left(y\left(t_{k}^{-}\right)\right)+\sum_{i=1}^{k-1} \prod_{j=1}^{k-i}\left(t_{k-j+1}-t_{k-j}\right)^{\alpha-1} I_{i}\left(y\left(t_{i}^{-}\right)\right)\right] \\
& +\frac{1}{\Gamma(\alpha)} \int_{t_{k}}^{t}(t-s)^{\alpha-1} v_{n}(s) d s
\end{aligned}
$$

We use the fact that $F$ has compact value and by passing (if necessary) onto a subsequence to obtain that $v_{n}$ converges to $v$ in $L^{1}([0, T], \mathbb{R})$, we get $v \in S_{F, y}$, and for each $t \in(0, T]$, we have

$$
\begin{aligned}
u_{n}(t) \rightarrow u(t)= & \left(t-t_{k}\right)^{\alpha-1} \prod_{i=1}^{k}\left(t_{i}-t_{i-1}\right)^{\alpha-1} c \\
& +\frac{\left(t-t_{k}\right)^{\alpha-1}}{\Gamma(\alpha)}\left[\int_{t_{k-1}}^{t_{k}}\left(t_{k}-s\right)^{\alpha-1} v(s) d s\right. \\
& \left.+\sum_{i=1}^{k-1} \prod_{j=1}^{k-i}\left(t_{k-j+1}-t_{k-j}\right)^{\alpha-1} \int_{t_{i-1}}^{t_{i}}\left(t_{i}-s\right)^{\alpha-1} v(s) d s\right] \\
& +\frac{\left(t-t_{k}\right)^{\alpha-1}}{\Gamma(\alpha)}\left[I_{k}\left(y\left(t_{k}^{-}\right)\right)+\sum_{i=1}^{k-1} \prod_{j=1}^{k-i}\left(t_{k-j+1}-t_{k-j}\right)^{\alpha-1} I_{i}\left(y\left(t_{i}^{-}\right)\right)\right] \\
& +\frac{1}{\Gamma(\alpha)} \int_{t_{k}}^{t}(t-s)^{\alpha-1} v(s) d s
\end{aligned}
$$

Hence, $u \in F(y)$.

Now, we will prove the existence of a real number $\delta<1$ for which

$$
H_{d}(F(x), F(y)) \leq \delta\|x-y\|_{P C_{*}}, \quad \text { for all } x, y \in P C_{*}([0, T], \mathbb{R})
$$

For each $x, y \in P C_{*}([0, T], \mathbb{R})$ and $h_{1} \in P(x)$, we can find $v_{1}(t) \in F(t, x(t))$ such that, for all $t \in(0, T]$,

$$
h_{1}(t)=\left(t-t_{k}\right)^{\alpha-1} \prod_{i=1}^{k}\left(t_{i}-t_{i-1}\right)^{\alpha-1} c
$$




$$
\begin{aligned}
& +\frac{\left(t-t_{k}\right)^{\alpha-1}}{\Gamma(\alpha)}\left[\int_{t_{k-1}}^{t_{k}}\left(t_{k}-s\right)^{\alpha-1} v_{1}(s) d s\right. \\
& \left.+\sum_{i=1}^{k-1} \prod_{j=1}^{k-i}\left(t_{k-j+1}-t_{k-j}\right)^{\alpha-1} \int_{t_{i-1}}^{t_{i}}\left(t_{i}-s\right)^{\alpha-1} v_{1}(s) d s\right] \\
& +\frac{\left(t-t_{k}\right)^{\alpha-1}}{\Gamma(\alpha)}\left[I_{k}\left(x\left(t_{k}^{-}\right)\right)+\sum_{i=1}^{k-1} \prod_{j=1}^{k-i}\left(t_{k-j+1}-t_{k-j}\right)^{\alpha-1} I_{i}\left(x\left(t_{i}^{-}\right)\right)\right] \\
& +\frac{1}{\Gamma(\alpha)} \int_{t_{k}}^{t}(t-s)^{\alpha-1} v_{1}(s) d s .
\end{aligned}
$$

From $\left(H_{3}\right)$, we have

$$
H_{d}(F(t, x(t)), F(t, y(t))) \leq p(t)|x(t)-y(t)| .
$$

Consequently, exists $w \in F(t, y(t))$ such that

$$
\left|v_{1}(t)-w\right| \leq p(t)|x(t)-y(t)|, \quad t \in(0, T] .
$$

Consider the map $U$ from $(0, T]$ into $\mathcal{P}(\mathbb{R})$ defined by

$$
U(t):=\left\{w(t) \in \mathbb{R}:\left|v_{1}(t)-w\right| \leq p(t)|x(t)-y(t)|\right\} .
$$

From [9, Proposition III. 4], the intersection between $U(t)$ and $F(t, y(t))$ is a measurable set. Therefore, we can find a measurable selection $v_{2}(\cdot)$ for $U(\cdot) \cap F(\cdot, y(\cdot))$. So, $v_{2}(t) \in F(t, y(t))$ and

$$
\left|v_{1}(t)-v_{2}(t)\right| \leq p(t)|x(t)-y(t)|, \quad \text { for all } 0<t \leq T .
$$

For every $0<t \leq T$, we define

$$
\begin{aligned}
h_{1}(t)= & \left(t-t_{k}\right)^{\alpha-1} \prod_{i=1}^{k}\left(t_{i}-t_{i-1}\right)^{\alpha-1} c \\
& +\frac{\left(t-t_{k}\right)^{\alpha-1}}{\Gamma(\alpha)}\left[\int_{t_{k-1}}^{t_{k}}\left(t_{k}-s\right)^{\alpha-1} v_{2}(s) d s\right. \\
& \left.+\sum_{i=1}^{k-1} \prod_{j=1}^{k-i}\left(t_{k-j+1}-t_{k-j}\right)^{\alpha-1} \int_{t_{i-1}}^{t_{i}}\left(t_{i}-s\right)^{\alpha-1} v_{2}(s) d s\right] \\
& +\frac{\left(t-t_{k}\right)^{\alpha-1}}{\Gamma(\alpha)}\left[I_{k}\left(y\left(t_{k}^{-}\right)\right)+\sum_{i=1}^{k-1} \prod_{j=1}^{k-i}\left(t_{k-j+1}-t_{k-j}\right)^{\alpha-1} I_{i}\left(y\left(t_{i}^{-}\right)\right)\right] \\
& +\frac{1}{\Gamma(\alpha)} \int_{t_{k}}^{t}(t-s)^{\alpha-1} v_{2}(s) d s .
\end{aligned}
$$

Thus,

$$
\begin{aligned}
& \left|\left(t-t_{k}\right)^{1-\alpha} h_{1}(t)-\left(t-t_{k}\right)^{1-\alpha} h_{2}(t)\right| \\
\leq & \frac{1}{\Gamma(\alpha)}\left[\int_{t_{k-1}}^{t_{k}}\left(t_{k}-s\right)^{\alpha-1}\left|v_{2}(s)-v_{1}(s)\right| d s\right.
\end{aligned}
$$




$$
\begin{aligned}
& \left.+\sum_{i=1}^{k-1} \prod_{j=1}^{k-i}\left(t_{k-j+1}-t_{k-j}\right)^{\alpha-1} \int_{t_{i-1}}^{t_{i}}\left(t_{i}-s\right)^{\alpha-1}\left|v_{2}(s)-v_{1}(s)\right| d s\right] \\
& +\frac{1}{\Gamma(\alpha)}\left[\left|I_{k}\left(y\left(t_{k}^{-}\right)\right)-I_{k}\left(x\left(t_{k}^{-}\right)\right)\right|+\sum_{i=1}^{k-1} \prod_{j=1}^{k-i}\left(t_{k-j+1}-t_{k-j}\right)^{\alpha-1}\left|I_{i}\left(y\left(t_{i}^{-}\right)\right)-I_{i}\left(x\left(t_{i}^{-}\right)\right)\right|\right] \\
& +\frac{\left(t-t_{k}\right)^{1-\alpha}}{\Gamma(\alpha)} \int_{t_{k}}^{t}(t-s)^{\alpha-1}\left|v_{2}(s)-v_{1}(s)\right| d s .
\end{aligned}
$$

Hence,

$$
\left\|h_{1}-h_{2}\right\|_{P C_{*}} \leq\left[\frac{T^{\alpha}\|p\|_{\infty} \Gamma(\alpha)\left(1+m T^{\alpha-1}\right)}{\Gamma(2 \alpha)}+\frac{m T_{0}^{\alpha-1} L}{\Gamma(\alpha)}\right]\|x-y\|_{P C_{*}},
$$

where $T_{0}=\min _{i=1, \ldots, m}\left(t_{i+1}-t_{i}\right)$. Interchange $x$ by $y$ in the previous computation, we obtain

$$
H_{d}(P(x), P(y)) \leq \delta\|x-y\|_{P C_{*}},
$$

where $\delta=\frac{T^{\alpha}\|p\|_{\infty} \Gamma(\alpha)\left(1+m T^{\alpha-1}\right)}{\Gamma(2 \alpha)}+\frac{m T_{0}^{\alpha-1} L}{\Gamma(\alpha)}<1$. Hence, $P$ is a contraction, and from Lemma 3.4, it has a fixed point $y$ considered as a solution of (1.1).

Step 2. Structure of the solutions set. Let

$$
S_{F}(c)=\left\{y \in P C_{*}([0, T], \mathbb{R}): y \text { is solution of }(1.1)\right\} .
$$

We will prove that $S_{F}(c)$ is compact in $P C_{*}([0, T], \mathbb{R})$. Let $\left\{y_{n}\right\}_{n \in \mathbb{N}} \in S_{F}(c)$, then there exists $v_{n} \in S_{F, y_{n}}$ and $t \in J$ such that

$$
\begin{aligned}
y_{n}(t)= & \left(t-t_{k}\right)^{\alpha-1} \prod_{i=1}^{k}\left(t_{i}-t_{i-1}\right)^{\alpha-1} c \\
& +\frac{\left(t-t_{k}\right)^{\alpha-1}}{\Gamma(\alpha)}\left[\int_{t_{k-1}}^{t_{k}}\left(t_{k}-s\right)^{\alpha-1} v_{n}(s) d s\right. \\
& \left.+\sum_{i=1}^{k-1} \prod_{j=1}^{k-i}\left(t_{k-j+1}-t_{k-j}\right)^{\alpha-1} \int_{t_{i-1}}^{t_{i}}\left(t_{i}-s\right)^{\alpha-1} v_{n}(s) d s\right] \\
& +\frac{\left(t-t_{k}\right)^{\alpha-1}}{\Gamma(\alpha)}\left[I_{k}\left(y\left(t_{k}^{-}\right)\right)+\sum_{i=1}^{k-1} \prod_{j=1}^{k-i}\left(t_{k-j+1}-t_{k-j}\right)^{\alpha-1} I_{i}\left(y\left(t_{i}^{-}\right)\right)\right] \\
& +\frac{1}{\Gamma(\alpha)} \int_{t_{k}}^{t}(t-s)^{\alpha-1} v_{n}(s) d s
\end{aligned}
$$

From $\left(H_{1}\right)$ and $\left(H_{2}\right)$, there exists $M_{1}>0$ such that $\left.\left\|y_{n}\right\|\right|_{P C_{*}} \leq M_{1}$ for every $n \geq 1$, and the set $\left\{y_{1}, y_{2}, \ldots, y_{n}, \ldots\right\}$ is equicontinuous in $P C_{*}([0, T], \mathbb{R})$. By using Theorem 3.1 , we can find a subsequence of $\left(y_{n}\right)$ (still denoted $\left(y_{n}\right)$ ) converges to $y$ in the space $P C_{*}([0, T], \mathbb{R}$. Now, we will prove the existence of $v(\cdot) \in F(\cdot, y(\cdot))$ and an element 
$t \in J$ such that

$$
\begin{aligned}
y(t)= & \left(t-t_{k}\right)^{\alpha-1} \prod_{i=1}^{k}\left(t_{i}-t_{i-1}\right)^{\alpha-1} c \\
& +\frac{\left(t-t_{k}\right)^{\alpha-1}}{\Gamma(\alpha)}\left[\int_{t_{k-1}}^{t_{k}}\left(t_{k}-s\right)^{\alpha-1} v(s) d s\right. \\
& \left.+\sum_{i=1}^{k-1} \prod_{j=1}^{k-i}\left(t_{k-j+1}-t_{k-j}\right)^{\alpha-1} \int_{t_{i-1}}^{t_{i}}\left(t_{i}-s\right)^{\alpha-1} v(s) d s\right] \\
& +\frac{\left(t-t_{k}\right)^{\alpha-1}}{\Gamma(\alpha)}\left[I_{k}\left(y\left(t_{k}^{-}\right)\right)+\sum_{i=1}^{k-1} \prod_{j=1}^{k-i}\left(t_{k-j+1}-t_{k-j}\right)^{\alpha-1} I_{i}\left(y\left(t_{i}^{-}\right)\right)\right] \\
& +\frac{1}{\Gamma(\alpha)} \int_{t_{k}}^{t}(t-s)^{\alpha-1} v(s) d s .
\end{aligned}
$$

Use the fact that $F(\cdot, \cdot)$ is upper semi-continuous, we can show that for every positive real number $\varepsilon$, there exists a positive integer $n_{0}$ such that

$$
v_{n}(t) \in F\left(t, y_{n}(t)\right) \subset F\left(t, y_{n}(t)\right)+\varepsilon B(0,1) \quad \text { a.e. } t \in J \text {, for every } n \geq n_{0} \text {. }
$$

Using the compactness of $F(\cdot, \cdot)$ we get the subsequence $v_{n m}(\cdot)$ such that

$$
v_{n m}(\cdot) \rightarrow v(\cdot) \text { and } \quad v(t) \in F(t, y(t)) \text { a.e. } t \in J .
$$

From $\left(H_{1}\right)$, we have

$$
v_{n m}(\cdot) \leq \bar{a} t^{\alpha-1} M_{1}+\bar{b} \quad \text { a.e. } t \in J .
$$

Using Lebesgue's dominated convergence theorem, we obtain that $v \in L^{1}(J, \mathbb{R})$, so $v \in S_{F, y}$. Therefore, for all $t \in J$

$$
\begin{aligned}
y(t)= & \left(t-t_{k}\right)^{\alpha-1} \prod_{i=1}^{k}\left(t_{i}-t_{i-1}\right)^{\alpha-1} c \\
& +\frac{\left(t-t_{k}\right)^{\alpha-1}}{\Gamma(\alpha)}\left[\int_{t_{k-1}}^{t_{k}}\left(t_{k}-s\right)^{\alpha-1} v(s) d s\right. \\
& \left.+\sum_{i=1}^{k-1} \prod_{j=1}^{k-i}\left(t_{k-j+1}-t_{k-j}\right)^{\alpha-1} \int_{t_{i-1}}^{t_{i}}\left(t_{i}-s\right)^{\alpha-1} v(s) d s\right] \\
& +\frac{\left(t-t_{k}\right)^{\alpha-1}}{\Gamma(\alpha)}\left[I_{k}\left(y\left(t_{k}^{-}\right)\right)+\sum_{i=1}^{k-1} \prod_{j=1}^{k-i}\left(t_{k-j+1}-t_{k-j}\right)^{\alpha-1} I_{i}\left(y\left(t_{i}^{-}\right)\right)\right] \\
& +\frac{1}{\Gamma(\alpha)} \int_{t_{k}}^{t}(t-s)^{\alpha-1} v(s) d s .
\end{aligned}
$$

Then $S_{F}(c)$ is compact. 
Let $f \in F$ be a function which is mLL. Consider the following single-valued problem

$$
\left\{\begin{array}{l}
{ }^{R L} D^{\alpha} y(t)=f(t, y(t)) \quad \text { a.e. } t \in J=(0, T], t \neq t_{k}, \\
\lim _{t \rightarrow 0^{+}} t^{1-\alpha} y(t)=c \\
\left.\Delta^{*} y\right|_{t_{k}}=I_{k}\left(y\left(t_{k}^{-}\right)\right) .
\end{array}\right.
$$

Using Banach fixed point theorem, we can prove easily that the problem (3.5) has a unique solution $\bar{x}$. Consider the homotopy $h: S_{F}(c) \times[0,1] \rightarrow S_{F}(c)$ defined by

$$
h(y, \lambda):= \begin{cases}y, & \text { for } \lambda=1 \text { and } y \in S_{F}(c), \\ \bar{x}, & \text { for } \lambda=0\end{cases}
$$

Note that

$$
h(y, \lambda)(t)= \begin{cases}y(t), & \text { for } 0<t \leq \lambda T \\ \bar{x}(t), & \text { for } \lambda T<t \leq T .\end{cases}
$$

We will show that $h$ is a continuous homotopy. Let $\left(y_{n}, \lambda_{n}\right) \in S_{F}(c) \times[0,1]$ such that $\left(y_{n}, \lambda_{n}\right) \rightarrow(y, \lambda)$. We shall show that $h\left(y_{n}, \lambda_{n}\right) \rightarrow h(y, \lambda)$. We have

$$
h\left(y_{n}, \lambda_{n}\right)(t)= \begin{cases}y_{n}(t), & \text { for } t \in\left(0, \lambda_{n} T\right], \\ \bar{x}(t), & \text { for }\left(\lambda_{n} T, T\right] .\end{cases}
$$

(a) If $\lim _{n \rightarrow \infty} \lambda_{n}=0$, then $H(y, 0)(t)=\bar{x}(t)$, for $t \in(0, T]$, hence

$$
\left\|H\left(y_{n}, \lambda_{n}\right)-H(y, \lambda)\right\|_{P C_{*}} \leq\left\|y_{n}-y\right\|_{P C_{*}}+\left\|y_{n}-\bar{x}\right\|_{\left[0, \lambda_{n} T\right]},
$$

which tends to 0 as $n \rightarrow+\infty$. The case $\lim _{n \rightarrow \infty} \lambda_{n}=1$ can be processed in the same way.

(b) If $\lambda_{n} \neq 0$ and $0<\lim _{n \rightarrow \infty} \lambda_{n}=\lambda<1$. We distinguish two sub-cases.

(i) Since $y_{n} \in S_{F}(c)$, there exists $v_{n} \in S_{F, y_{n}}$ such that for $t \in\left(0, \lambda_{n} T\right]$

$$
\begin{aligned}
y_{n}(t)= & \left(t-t_{k}\right)^{\alpha-1} \prod_{i=1}^{k}\left(t_{i}-t_{i-1}\right)^{\alpha-1} c \\
& +\frac{\left(t-t_{k}\right)^{\alpha-1}}{\Gamma(\alpha)}\left[\int_{t_{k-1}}^{t_{k}}\left(t_{k}-s\right)^{\alpha-1} v_{n}(s) d s\right. \\
& \left.+\sum_{i=1}^{k-1} \prod_{j=1}^{k-i}\left(t_{k-j+1}-t_{k-j}\right)^{\alpha-1} \int_{t_{i-1}}^{t_{i}}\left(t_{i}-s\right)^{\alpha-1} v_{n}(s) d s\right] \\
& +\frac{\left(t-t_{k}\right)^{\alpha-1}}{\Gamma(\alpha)}\left[I_{k}\left(y_{n}\left(t_{k}^{-}\right)\right)+\sum_{i=1}^{k-1} \prod_{j=1}^{k-i}\left(t_{k-j+1}-t_{k-j}\right)^{\alpha-1} I_{i}\left(y_{n}\left(t_{i}^{-}\right)\right)\right] \\
& +\frac{1}{\Gamma(\alpha)} \int_{t_{k}}^{t}(t-s)^{\alpha-1} v_{n}(s) d s .
\end{aligned}
$$

Since $y_{n} \rightarrow y$ as $n \rightarrow \infty$, there exists a positive real number $r>0$ such that $\left\|y_{n}\right\|_{P C_{*}} \leq r$, and as the function $F(\cdot, \cdot)$ is upper semi-continuous, for every 
positive real number $\varepsilon$, there exists a positive integer $n_{0}$ such that for every $n \geq n_{0}$, we have

$$
v_{n}(t) \in F\left(t, y_{n}(t)\right) \subset F\left(t, y_{n}(t)\right)+\varepsilon B(0,1) \quad \text { a.e. } t \in J .
$$

Using the fact that $F(\cdot, \cdot)$ has compact values, we can obtain the subsequence $v_{n m}(\cdot)$ such that

$$
v_{n m}(\cdot) \rightarrow v(\cdot) \quad \text { and } \quad v(t) \in F(t, y(t)) \quad \text { a.e. } t \in J .
$$

From $\left(H_{1}\right)$, we have

$$
v_{n m}(\cdot) \leq \bar{a} t^{\alpha-1} M_{1}+\bar{b} \quad \text { a.e. } t \in J .
$$

Using Lebesgue's dominated convergence theorem, we get $v \in L^{1}(J, \mathbb{R})$, so $v \in S_{F, y}$. Since $I_{k}$ are continuous functions, then, for all $t \in J$, we get

$$
\begin{aligned}
y(t)= & \left(t-t_{k}\right)^{\alpha-1} \prod_{i=1}^{k}\left(t_{i}-t_{i-1}\right)^{\alpha-1} c \\
& +\frac{\left(t-t_{k}\right)^{\alpha-1}}{\Gamma(\alpha)}\left[\int_{t_{k-1}}^{t_{k}}\left(t_{k}-s\right)^{\alpha-1} v(s) d s\right. \\
& \left.+\sum_{i=1}^{k-1} \prod_{j=1}^{k-i}\left(t_{k-j+1}-t_{k-j}\right)^{\alpha-1} \int_{t_{i-1}}^{t_{i}}\left(t_{i}-s\right)^{\alpha-1} v(s) d s\right] \\
& +\frac{\left(t-t_{k}\right)^{\alpha-1}}{\Gamma(\alpha)}\left[I_{k}\left(y\left(t_{k}^{-}\right)\right)+\sum_{i=1}^{k-1} \prod_{j=1}^{k-i}\left(t_{k-j+1}-t_{k-j}\right)^{\alpha-1} I_{i}\left(y\left(t_{i}^{-}\right)\right)\right] \\
& +\frac{1}{\Gamma(\alpha)} \int_{t_{k}}^{t}(t-s)^{\alpha-1} v(s) d s .
\end{aligned}
$$

(ii) Since $t \in\left(\lambda_{n} T, T\right]$, then

$$
h\left(y_{n}, \lambda_{n}\right)(t)=h(y, \lambda)(t)=\bar{x} .
$$

Thus,

$$
\left\|h\left(y_{n}, \lambda_{n}\right)-h(y, \lambda)\right\|_{P C_{*}} \rightarrow 0 \quad n \rightarrow \infty .
$$

Consequently, $h$ is continuous, and hence, $S_{F}(c)$ is contractible to the point $\bar{x}$. Therefore, $S_{F}(c)$ is an acyclic space.

Example 3.1 (An application). Consider

$$
\left\{\begin{array}{l}
R L D^{\frac{1}{2}} y(t) \in F(t, y(t)) \quad \text { a.e. } t \in(0,1], t \neq \frac{1}{2}, \\
\lim _{t \rightarrow 0^{+}} t^{\frac{1}{2}} y(t)=\frac{1}{4}, \\
\left.\Delta^{*} y\right|_{t=\frac{1}{2}}=\frac{1}{9}\left|\sin \left(y\left(\frac{1}{2}^{-}\right)\right)\right|+1,
\end{array}\right.
$$


where $T=1, m=1, t_{1}=\frac{1}{2}$,

$$
F(t, x)=\left[0, \frac{1}{9} \sin x+\frac{|x|}{t+9}+\frac{1}{9}\right]
$$

and

Clearly,

$$
I_{1}(u)=\frac{1}{9}|\sin (u)|+1, \quad \text { for } u \in \mathbb{R}
$$

$$
\begin{aligned}
\sup _{v \in F(t, x)}|v| & \leq \frac{1}{9}+\frac{1}{9}|\sin x|+\frac{|x|}{t+9}, \\
H_{d}(F(t, x), F(t, y)) & \leq\left[\frac{1}{t+9}+\frac{1}{9}\right]|x-y|
\end{aligned}
$$

and

$$
\left|I_{1}(u)\right| \leq \frac{1}{9}|\sin (u)|+1, \quad\left|I_{1}(u)-I_{1}(v)\right| \leq \frac{1}{9}|u-v| .
$$

Let $p(t)=\frac{1}{9}+\frac{1}{t+9}$. Then $\|p\|_{\infty}=\frac{2}{9}$ and

$$
\frac{T^{\alpha}\|p\|_{\infty} \Gamma(\alpha)\left(1+m T^{\alpha-1}\right)}{\Gamma(2 \alpha)}+\frac{m T_{0}^{\alpha-1} L}{\Gamma(\alpha)} \simeq 0,73965<1 .
$$

Theorem 3.2 confirms that (3.6) has at least one solution. In addition, it is clear that $F$ is a mLL-selectionable multivalued map (i.e., the function $f(t, u)=\frac{1}{9} \sin u+\frac{|u|}{t+9}+\frac{1}{9}$ is measurable, locally-Lipchitzian) with compact and convex values. Consequently, the solution set is contractible and compact, and hence it is acyclic.

Acknowledgements. I would like to thank the editor and referees for his/her suggestions that improved the quality of the paper. Also I thank my PhD supervisor Pr. Hedia Benaouda for his helpful comments and encouragement.

\section{REFERENCES}

[1] S. Abbes and M. Benchohra, Advanced Functional Evolution Equations and Inclusions, Springer, New York, London, 2015.

[2] R. P. Agarwal, M. Benchohra and S. Hamani, A survey on existence results for boundary value problems of nonlinear fractional differential equations and inclusions, Acta Appl. Math. 109 (2010), 973-1033.

[3] R. P. Agarwal, M. Benchohra, J. Nieto and A. Ouahab, Some results for integral inclusions of Volterra type in Banach spaces, Adv. Difference Equ. (2010), DOI 10.1155/2010/798067.

[4] Z. Agur, L. Cojocaru, G. Mazaur, R. M. Anderson and Y. L. Danon, Pulse mass measles vaccination across age cohorts, Proc. Nat. Acad. Sci. USA 90 (1993), 11698-11702.

[5] J. Andres and L. Górniewicz, Topological Principles for Boundary Value Problems, Kluwer, Dordrecht, 2003.

[6] J. P. Aubin and A. Cellina, Differential Inclusions, Springer-Verlag, New York, 1984.

[7] J. P. Aubin and H. Frankowska, Set-Valued Analysis, Birkhauser, Boston, 1990. 
[8] M. Benchohra, J. Nieto and A. Ouahab, Impulsive differential inclusions involving evolution operators in separable Banach spaces, Ukrainian Math. J. 64 (2012), 991-1018.

[9] C. Castaing and M. Valadier, Convex Analysis and Measurable Multifunctions, Lecture Notes in Mathematics 580, Springer-Verlag, Berlin, Heidelberg, New York, 1977.

[10] Y. Chalco-Cano, J. J. Nieto, A. Ouahab and H. Román-Flores, Solution set for fractional differential equations with Riemann-Liouville derivative, Fract. Calc. Appl. Anal. 16 (2013), 682-694.

[11] H. Covitz and S. B. Nadler, Multivalued contraction mappings in generalized metric spaces, Israel J. Math. 8 (1970), 5-11.

[12] K. Deimling, Multivalued Differential Equations, De Gruyter, Berlin, New York, 1992.

[13] D. Delbosco and L. Rodino, Existence and uniqueness for a nonlinear fractional differential equation, J. Math. Anal. Appl. (1996), 609-625.

[14] K. Djebali, L. Górniewicz and A. Ouahab, Solutions Sets for Differential Equations and Inclusions, De Gruyter Series in Nonlinear Analysis and Applications 18, de Gruyter, Berlin, 2013.

[15] S. Djebali, L. Górniewicz and A. Ouahab, Existence and Structure of Solution Sets for Impulsive Differential Inclusions, a Survey, Lecture Notes in Nonlinear Analysis 13, Nicolaus Copernicus University, Juliusz Schauder Center for Nonlinear Studies, Torun, 2012.

[16] S. Djebali, L. Górniewicz and A. Ouahab, Topological structure of solution sets for impulsive differential inclusions in Fréchet spaces, Nonlinear Anal. 74 (2011), 2141-2169.

[17] R. Dragoni, P. Nistri, P. Zeccaand and J. W. Macki, Solution Sets of Differential Equations in Abstract Spaces, Longman, Edinburgh, 1996.

[18] A. M. A. El-Sayed, Nonlinear functional differential equations of arbitrary orders, Nonlinear Anal. 33 (1998), 181-186.

[19] A. M. A. El-Sayed and A. G. Ibrahim, Multivalued fractional differential equations, Appl. Math. Comput. 68 (1995), 15-25.

[20] L. Gaul, P. Klein and S. Kempfle, Damping description involving fractional operators, Mechanical Systems and Signal Processing 5 (1991), 81-88.

[21] W. G. Glockle and T. F. Nonnenmacher, A fractional calculus approach of self-similar protein dynamics, Biophysical Journal 68 (1995), 46-53.

[22] L. Górniewicz, On the solution sets of differential inclusions, J. Math. Anal. Appl. (1986), 235-244.

[23] L. Górniewicz, Topological Fixed Point Theory of Multivalued Mappings, Mathematics and its Applications 495, Kluwer Academic Publishers, Dordrecht, 1999.

[24] A. Granas and J. Dugundji, Fixed Point Theory, Springer-Verlag, New York, 2003.

[25] A. Grudzka and S. Ruszkowski, Structure of the solutions set to differential inclusions with impulses and variable times, Electronic J. Diff. Equ. (2015), 1-16.

[26] J. Henderson and A. Ouahab, Impulsive differential inclusions with fractional order, Comput. Math. Appl. 59 (2010), 1191-1226.

[27] S. Hu and N. S. Papageorgiou, Handbook of Multi-Valued Analysis, Volume I: Theory, Kluwer, Dordrecht, 1997.

[28] S. Hu and N. S. Papageorgiou, Handbook of Multi-Valued Analysis Volume II: Applications, Kluwer, Dordrecht, 2000.

[29] M. Kamenskii, V. Obukhovskii and P. Zecca, Condensing Multivalued Maps and Semilinear Differential Inclusions in Banach Spaces, Nonlinear Analysis and Applications 7, Walter de Gruyter, Berlin, New York, 2001.

[30] E. Krüger-Thiemer, Formal theory of drug dosage regimens. I, J. Theoret. Biol. 13 (1966), 212-235.

[31] E. Krüger-Thiemer, Formal theory of drug dosage regimens: II. The exact plateau effect, J. Theoret. Biol. 23 (1969), 169-190. 
[32] A. Lasota and Z. Opial, An application of the Kakutaniky fan theorem in the theory of ordinary differential equations, Bull. Acad. Pol. Sci. Ser. Sci. Math. Astronom. Phys. 13 (1965), 781-786.

[33] J. M. Lasry and R. Robert, Analyse Non Lineaire Multivoque, Centre de Recherche de Mathématique de la Décision, Université de Dauphine, Paris, CNRS, 1976.

[34] F. Metzler, W. Schick, H. G. Kilian and T. F. Nonnenmacher, Relaxation in filled polymers, a fractional calculus approach, The Journal of Chemical Physics (1995), 7180-7186.

[35] J. Musielak, Introduction to Functional Analysis, PWN, Warsaw, 1976.

[36] D. Wagner, Survey of measurable selection theorems, SIAM J. Control Optim. 15 (1977), 859903.

[37] Z. Yong, W. Rong-Nian and P. Li, Topological Structure of the Solution Set for Evolution Inclusions, Springer-Verlag, New York, 2017.

[38] S. Zhang, Positive solutions for boundary-value problems of nonlinear fractional diffrential equations, Electron. J. Differential Equations 36 (2006), 1-12.

[39] Y. Zhou, Basic Theory of Fractional Differential Equations, World Scientific, Singapore, 2014.

[40] Y. Zhou, Fractional Evolution Equations and Inclusions, Analysis and Control, Elsevier, Amsterdam, 2015.

${ }^{1}$ Department of MAThematics,

Djillali liabes University of Sidi Bel-AbBès,

P.O. Box 89, Sidi Bel-AbBès 22000 , Algeria

Email address: beddani2004@yahoo.fr 